This item was submitted to Loughborough's Research Repository by the author.

Items in Figshare are protected by copyright, with all rights reserved, unless otherwise indicated.

Shape and wobbling wave excitations in Josephson junctions: exact solutions of the (2+1)-dimensional sine-Gordon model

PLEASE CITE THE PUBLISHED VERSION

http://dx.doi.org/10.1103/PhysRevB.80.094509

PUBLISHER

(C) American Physical Society

VERSION

VoR (Version of Record)

LICENCE

CC BY-NC-ND 4.0

REPOSITORY RECORD

Gulevich, Dmitry R., F.V. Kusmartsev, Sergey Savel'ev, V.A. Yampol'skii, and Franco Nori. 2019. "Shape and Wobbling Wave Excitations in Josephson Junctions: Exact Solutions of the (2+1)-dimensional Sine-gordon Model". figshare. https://hdl.handle.net/2134/12762. 


\title{
Shape and wobbling wave excitations in Josephson junctions: Exact solutions of the $(2+1)$-dimensional sine-Gordon model
}

\author{
D. R. Gulevich, ${ }^{1,2}$ F. V. Kusmartsev, ${ }^{1}$ Sergey Savel'ev, ${ }^{1,2}$ V. A. Yampol'skii, ${ }^{2,3}$ and Franco Nori ${ }^{2,4}$ \\ ${ }^{1}$ Physics Department, Loughborough University, Leicestershire LE11 3TU, United Kingdom \\ ${ }^{2}$ Advanced Science Institute, The Institute of Physical and Chemical Research (RIKEN), Wako-shi, Saitama 351-0198, Japan \\ ${ }^{3}$ Usikov Institute for Radiophysics and Electronics, Ukrainian Academy of Science, 61085 Kharkov, Ukraine \\ ${ }^{4}$ Department of Physics, Center for Theoretical Physics, University of Michigan, Ann Arbor, Michigan 48109, USA
}

(Received 15 April 2009; revised manuscript received 3 August 2009; published 15 September 2009)

\begin{abstract}
We predict a class of excitations propagating along a Josephson vortex in two-dimensional Josephson junctions. These excitations are associated with the distortion of a Josephson vortex line of an arbitrary profile. We derive a universal analytical expression for the energy of arbitrary-shape excitations, investigate their influence on the dynamics of a vortex line, and discuss conditions where such excitations can be created. Finally, we show that such excitations play the role of a clock for a relativistically-moving Josephson vortex and suggest an experiment to measure a time-dilation effect analogous to that in special relativity. The position of the shape excitation on a Josephson vortex acts like a "minute hand" showing the time in the rest frame associated with the vortex. Remarkably, at some conditions, the shape wave can carry negative energy: a vortex with the shape excitation can have less energy than the same vortex without it.
\end{abstract}

DOI: 10.1103/PhysRevB.80.094509

PACS number(s): 74.50.+r, 05.45.Yv, 03.75.Lm

\section{INTRODUCTION}

The sine-Gordon (SG) model describes a variety of physical systems, including ferromagnets, information transport in microtubules, dislocations in crystals, nonlinear optics, Josephson junctions, and high-temperature superconductors (see, e.g., Refs. 1-4). The (1+1)D SG model is fully integrable and possesses well-known exact solutions in the form of solitons and breathers. ${ }^{3,4}$

In this work, we use the standard notation " $(1+1) \mathrm{D}$," rather than "two-dimensional (2D)," to indicate that one of the dimensions is spatial and the second one is temporal; this is needed to avoid confusing this $(1+1) \mathrm{D}$ model with the stationary $2 \mathrm{D}$ case where $2 \mathrm{D}$ refers to two spatial dimensions.

Depending on the physical realization, solitons may describe different objects, for instance, magnetic domain walls in ferromagnets or fluxons in Josephson junctions. There exist mathematical techniques that allow constructing solutions of the $(1+1) \mathrm{D}$ SG, e.g., such as the Bäcklund transformation, the Hirota, and inverse scattering methods (see, e.g., Refs. 3 and 4). Although real systems described by the SG model may contain additional terms associated with damping or external conditions, such terms can be taken into account as perturbations to the exact solutions. ${ }^{5,6}$

To describe more general systems, one should depart from the well-studied $(1+1) \mathrm{D}$ model and deal with the $(2+1) \mathrm{D}$ SG equation, which is no longer fully integrable. The extra dimension opens new avenues for the existence of phenomena which are absent in the one-dimensional (1D) case (see, e.g., Refs. 7 and 8). Different kinds of exact solutions of the $2+1$ dimensional sine-Gordon system have been given by many authors. ${ }^{9}$ The analysis of symmetries in the sineGordon system can help to find these solutions and in some cases lead to solutions involving arbitrary functions. ${ }^{10,11}$

Here we show that there exists a class of exact solutions of the $(2+1) \mathrm{D}$ SG equation which describes the propagation of excitations of an arbitrary shape along a Josephson vortex. The property of these excitations such as transmitting pulses of electromagnetic radiation of arbitrary shape along a Josephson vortex could be useful for the transmission of information in various high-frequency Josephson devices (see, e.g., the theoretical and experimental works in Refs. 12-14).

Experiments ${ }^{15}$ have shown that Josephson vortices can exhibit Lorentz contraction. However, so far, another relativistic effect, time dilation, has been considered difficult to detect in Josephson systems. In order to observe the time dilation, an internal degree of freedom is needed to act as a clock. However, in $(1+1) \mathrm{D}$ such clock is absent because solitons of the $(1+1)$ D SG equation do not possess internal oscillation modes. Recently, time dilation was proposed ${ }^{16}$ in junctions obeying the double sine-Gordon equation. There, an internal degree of freedom of a vortex, due to a special feature of the double SG model, was proposed as a clock for a moving vortex. Here we show that there is no need to deviate from the original pure sine-Gordon nonlinearity because an additional degree of freedom, due to the extra dimension, can be used to realize a clock. Thus, a conventional Josephson junction obeying the $(2+1)$ D SG can be used. Apart from the mathematical beauty of the solutions found, these might be used for information transmission and in digital superconducting systems based on conventional Josephson junctions or high-temperature superconductors. The solutions describing propagating electromagnetic waves and excitations in superconducting Josephson junctions are of potential practical importance. For example, recently a prototype analog-to-digital converter was developed in Ref. 17 suggesting that superconducting systems can be of future use for such applications as wireless communications, radars, switching networks, and $\mathrm{THz}$ technology. Moreover, integrated sub-THz receivers based on long Josephson junctions have been recently developed. ${ }^{13}$ We hope that the remarkable property of the solutions found here, such as guiding pulses of electromagnetic radiation of arbitrary shape, apart from 
their fundamental interest, could be used in future information processing technology.

Preliminary results on this problem have been presented in Refs. 11 and 14. This paper is far more complete version where we extend previous work and present recent unreported results. In Sec. II we present the concept of shape waves and give their physical interpretation as propagating distortions of a Josephson vortex line. Section III deals with solitary waves and investigate the effect of the Lorentz transformation. In Sec. IV we study shape waves in the form of harmonic sine-type distortions. In Sec. V we discuss kink shape excitations and propose possible experiments. In Sec. VI we introduce wobbling waves. Section VII is a concluding section where we discuss the main results of this work.

\section{SHAPE WAVES ON A JOSEPHSON VORTEX}

To illustrate the properties of the class of solutions studied here, we consider the $(2+1) \mathrm{D}$ SG equation,

$$
\varphi_{t t}-\varphi_{x x}-\varphi_{y y}+\sin \varphi=0 .
$$

For the case of a Josephson junction, $\varphi$ is the superconducting phase difference across the Josephson junction, the coordinates $x$ and $y$ are normalized by the Josephson penetration length $\lambda_{J}$, and the time $t$ is normalized by the inverse Josephson plasma frequency $\omega_{p}^{-1}$, see, e.g., Ref. 2 . The equation for the model of a 2D Josephson junction normally includes perturbation terms, such as different types of damping, bias, and external parameters. Here, we consider the basic model without damping or external parameters. This basic model must be studied first, which is the focus of this work, before attempting to consider the effects of additional terms, which is outside the scope of this work. These additional terms can be taken into account as perturbations to Eq. (1) in a manner similar to that developed by McLaughlin and Scott. ${ }^{5}$ In the case of a Josephson junction, the solitons of the SG equation describe vortices of superconducting current, called Josephson vortices or fluxons. A special class of solutions of the $(2+1) D$ SG equation can be constructed by Lorentz transforming the solutions of a stationary 2D SG equation. However, there are numerical solutions of the $(2+1) \mathrm{D}$ SG equation which cannot be derived from the $(1+1) \mathrm{D}$ model, e.g., radial breathers or pulsons. ${ }^{7}$

Here we are interested in the class of solutions of the $(2+1) \mathrm{D}$ SG equation of the following general form

$$
\varphi(x, y, t)=4 \arctan \exp [y-f(x \pm t)]
$$

which satisfies Eq. (1) exactly with an arbitrary real-valued twice-differentiable function $f(\xi)$, see Appendix A. The excitations, described by $f$, are associated with a local shift of a Josephson vortex in the transverse direction and have analogies with elastic shear waves in, e.g., solid mechanics and seismology. ${ }^{18}$ Such distortions propagate along the stationary vortex line with the fixed speed $|u|=1$ in units of the Swihart velocity $\bar{c}=\lambda_{J} \omega_{p}$. Hereafter, we will refer to these arbitraryshape excitations described by $f$ as shape waves.

Defining the total energy of a shape excitation as the difference of the energy of the solution Eq. (2) and the energy of the vortex without excitation $(f=0)$, we obtain an expres-

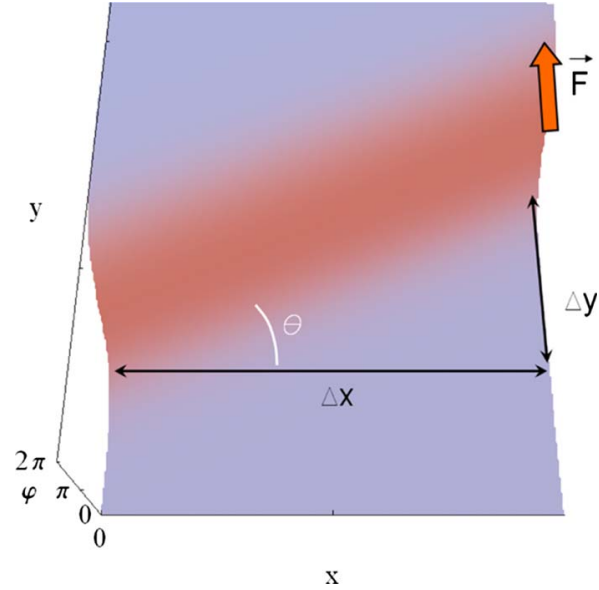

FIG. 1. (Color online) Figure 1 explains the shear modulus of a Josephson vortex. A vortex is shown along the $x$ direction, in 0 $\leq x \leq \Delta x$, and a transverse force $F$ is applied to the right end on the vortex segment shown. This perpendicular force $F$ causes a shear.

sion for the total energy $\Delta E$ as a functional of $f$

$$
\Delta E[f]=8 \int f^{\prime}(x)^{2} d x>0
$$

that is, a sum of the potential and kinetic energies of a shape excitation.

As in solid mechanics (e.g., Ref. 18), we can derive the shear modulus, equal to 8 per single Josephson vortex. Consider the part of a vortex in $0 \leq x \leq \Delta x$ lying along the $x$ axis and experiencing a transverse force $F$ acting on its right end, as shown in Fig. 1. Assume the boundary condition (on the side of the box in Fig. 1) where $\varphi(0, y)$ is fixed and equal to $\varphi(0, y)=4 \arctan \exp (y)$. The force causes a shear deformation $\Delta y$, so that on the right boundary

$$
\varphi(\Delta x, y)=4 \arctan \exp (y-\Delta y)
$$

and, therefore, in the limit $\Delta x \rightarrow 0$,

$$
\varphi(x, y)=4 \arctan \exp (y-x \tan \theta),
$$

where $\tan \theta=\Delta x / \Delta y$, see Fig. 1. Let us define the shear modulus $G$ for a single vortex as

$$
G=\frac{F}{\tan \theta}
$$

for $\Delta x \rightarrow 0$ and $\tan \theta$ being a measure of the deformation caused by the force $F$. The energy of the shear deformation [that is, a potential equal to exactly half of the expression for the total energy Eq. (17)],

$$
E[x \tan \theta]=4 \tan ^{2} \theta \Delta x=4 \frac{\Delta y^{2}}{\Delta x}
$$

and the restoring force is

$$
-\frac{d}{d(\Delta y)} E[x \tan \theta]=-8 \frac{\Delta y}{\Delta x}=-8 \tan \theta .
$$

If the force $F$ causes a deformation $\tan \theta$, in equilibrium $F$ $=8 \tan \theta$ and the shear modulus of a single vortex becomes 


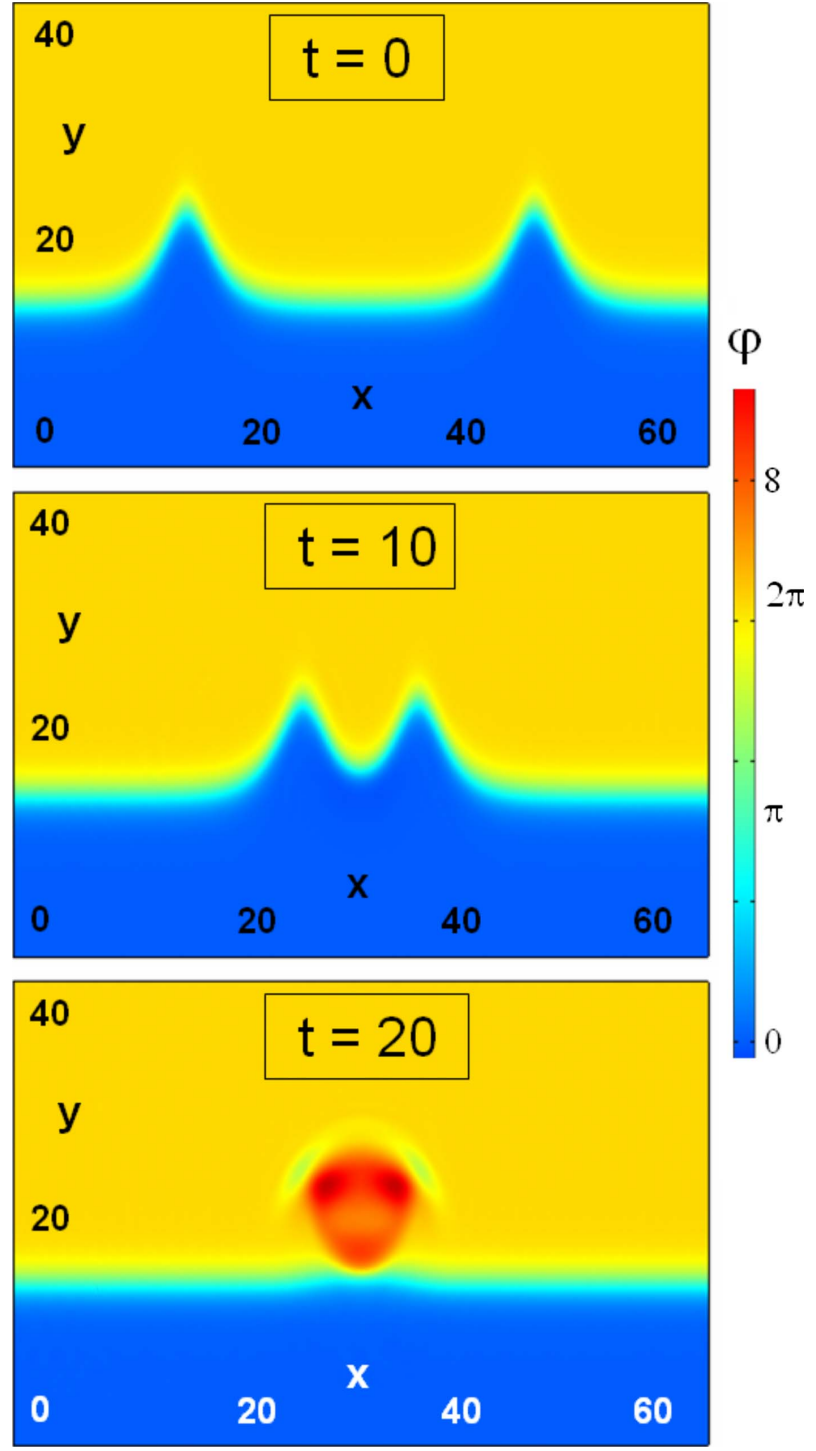

FIG. 2. (Color online) Collision and annihilation of two largeamplitude solitary waves, Eq. (2), with $f=8 / \cosh \left[\left(x-x_{0} \pm t\right) / 2\right]$ propagating along a Josephson vortex line. The color scale shows the superconducting phase difference $\varphi$. The figure shows $\varphi(x, y, t)$ snapshots obtained by numerically solving the $(2+1)$ D SG equation on a region of size $60 \times 40 \lambda_{J}^{2}$ with Neumann boundary conditions, $\mathbf{n} \cdot \nabla \varphi=0$. Initially, the two solitary waves keep their shape while propagating but their collision at $t \sim 15$ leads to their mutual annihilation and radiation of energy in all directions. Contrary to the large-amplitude waves shown here, small-amplitude solitary waves satisfying $\left|f^{\prime}(x+t) f^{\prime}(x-t)\right| \ll 1$, interact weakly during the collision and behave similarly to solitons.

$$
G=8 \quad \text { (per vortex). }
$$

As shown, one can introduce a shear modulus $G$ for a Josephson vortex. From this, the velocity of waves on a stationary vortex follows from the known formula

$$
c=\sqrt{\frac{G}{\rho}}=1,
$$

which is in agreement with Eq. (2).

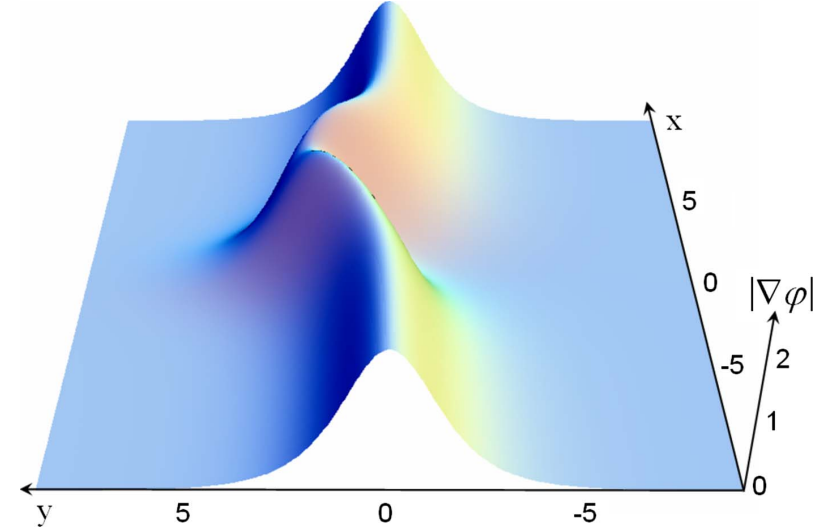

FIG. 3. (Color online) Snapshot at $t=0$ of a shape wave propagating along a Josephson vortex line according to Eq. (2) with $f$ $=2 / \cosh [(x \pm t) / 2]$. The gradient of the superconducting phase $|\Delta \varphi|$, which shows the distribution of the magnetic field, is plotted on the vertical axis.

\section{SOLITARY SHAPE WAVES}

Because of the arbitrariness of $f$, Eq. (2) describes a variety of excitations of different shapes. Choosing $f$ localized in a finite area, e.g., $f=A / \cosh (x-t)$, Eq. (2) describes an excitation, localized along $x$, that keeps its shape when propagating, i.e., a solitary wave, see Figs. 2 and $3 .{ }^{4}$ For each solitary wave of this type, there exists an antipartner with an $f$ of opposite sign in Eq. (2).

For solitary waves to be solitons, there is an additional important criterion restoring their shapes after they collide. Consider a trial function

$$
\varphi(x, y, t)=4 \arctan \exp [y-f(x+t) \pm f(x-t)]
$$

which for $t<0,|t| \ll 1$ describes two solitary shape waves moving toward each other. One can see that Eq. (2) can only approximately satisfy Eq. (1) when $\left|f^{\prime}(x+t) f^{\prime}(x-t)\right| \ll 1$ for all values of $x$ and $t$. This suggests that, in general, the condition for restoring the shapes may not be satisfied. Indeed, our numerical solutions of the $(2+1)$ D SG equation show that the waves keep their shapes when propagating but their collision can be destructive. The collision of two waves of large amplitudes may lead to their mutual annihilation, dissipating energy away from the vortex, see Fig. 2. However, smaller-amplitude waves behave similarly to solitons, dissipating very small energy and keeping their original shapes after collision.

The arbitrariness of $f(\xi)$ can be used to construct excitations of any desired shape. As an example, Fig. 4 shows numerical results with initial excitations having a complex profile. We consider a vortex and an antivortex which are pinned at some distance from each other. The excitations propagate along the vortex and antivortex in the same direction. Since solitary waves distort when colliding with each other, their interaction with a boundary has also a destructive effect: it can be seen as an interaction with the corresponding image behind the boundary. 


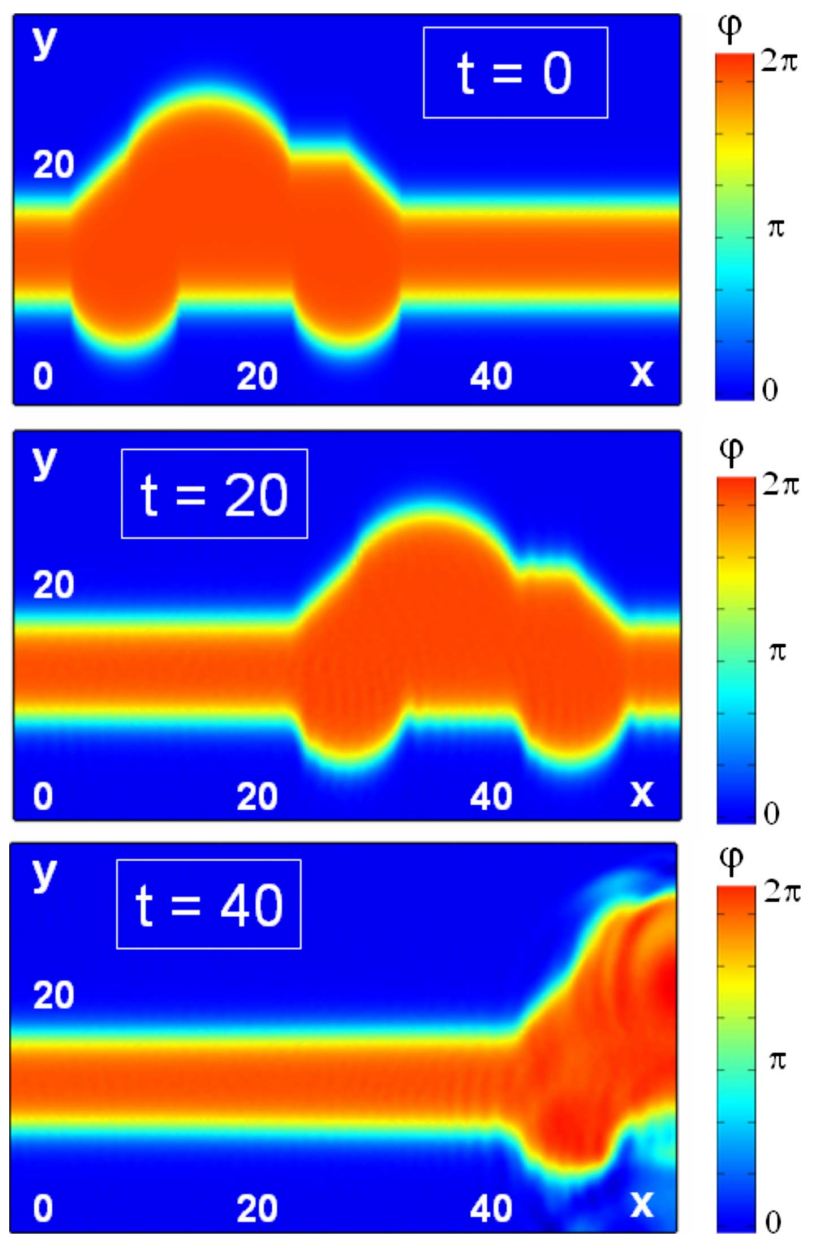

FIG. 4. (Color online) Solitary waves having any arbitrary initial form can be constructed using the solution Eq. (2) of the (2 $+1) \mathrm{D}$ SG equation. The color scale shows the superconducting phase difference $\varphi$. The figure shows $\varphi(x, y, t)$ snapshots obtained by numerically solving the $(2+1) \mathrm{D} S \mathrm{~S}$ equation on a region of size $60 \times 35 \lambda_{J}^{2}$ with Neumann boundary conditions, $\mathbf{n} \cdot \nabla \varphi=0$. The lower (green online) stripe represents a Josephson vortex while the top stripe is an antivortex pinned at a certain distance away from its partner. Solitary waves keep any arbitrary given shape while propagating, but their interaction with boundaries is destructive, as seen in the bottom frame where the solitary waves get significantly distorted by the boundary and unable to restore the original shape after the collision.

Figure 4 shows our numerical calculations when the two vortices were initially placed apart a distance much larger than the Josephson penetration length $\lambda_{J}$. We have not introduced any pinning sites explicitly because the interaction between vortices decreases exponentially on a scale $\lambda_{J}$ and in our case this does not influence the dynamics of the shape excitations.

Since the Eq. (1) is Lorentz invariant, one can obtain other solutions performing Lorentz transformations on Eq. (2). A Lorentz transformation along the $x$ axis leads to a Lorentz contraction of the excitation, but does not lead to any solution outside the class of Eq. (2), only rescaling the arbitrary function $f(\xi)$. The Lorentz transformation along $y$,
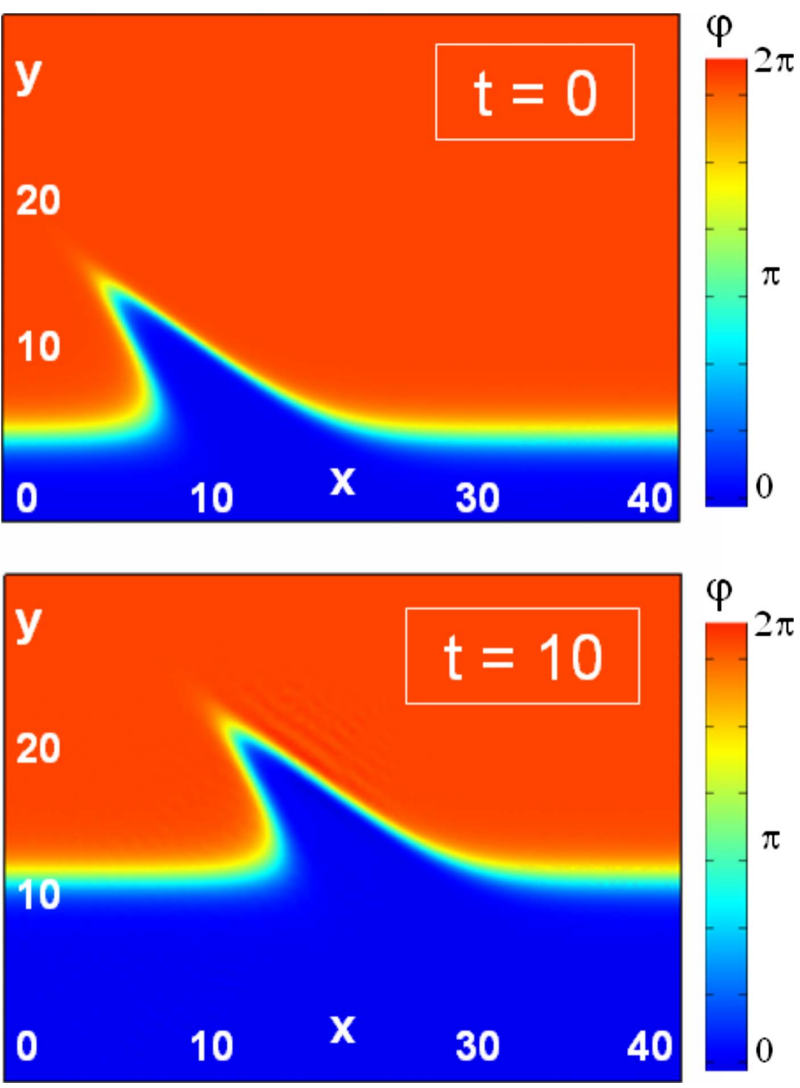

FIG. 5. (Color online) Solitary wave excitation $f(z)$ $=12 / \cosh (z / 2)$ propagating along a Josephson vortex moving with velocity $v=0.7$. The figure represents results of numerical calculations with a $(2+1)$ D SG equation in a box of the size $40 \times 30$ Josephson penetration lengths. The color scale in this figure denotes the superconducting phase difference $\varphi$.

$$
\left\{\begin{array}{l}
x \rightarrow x^{\prime}=x \\
y \rightarrow y^{\prime}=\frac{y-v t}{\sqrt{1-v^{2}}} \\
t \rightarrow t^{\prime}=\frac{t-v y}{\sqrt{1-v^{2}}},
\end{array}\right.
$$

turns out to be far more interesting, leading to a class of solutions more general than Eq. (2). Applying such transformation on Eq. (2), we obtain a solution of the $(2+1) \mathrm{D} \mathrm{SG}$ equation

$\varphi(x, y, t)=4 \arctan \exp \left[\frac{y-v t}{\sqrt{1-v^{2}}}-f\left(x \pm \frac{t-v y}{\sqrt{1-v^{2}}}\right)\right]$,

which describes a Josephson vortex line moving with velocity $v$ satisfying $-1<v<1$ and a propagating shape excitation described by $f(\xi)$. The $y$ dependence of the function $f(\xi)$ is intriguing: for nonzero $v$ the excitation profile becomes unusually tilted with respect to the vortex line, as illustrated in Fig. 5.

We will show that, apart from the Lorentz contraction, ${ }^{15}$ a propagating Josephson vortex exhibits time dilation which influences the dynamics of its shape excitations in Eq. (4). 
Recently, the Lorentz time dilation of a bound half-fluxon pair has been numerically studied ${ }^{16}$ for a long Josephson junction with a ferromagnetic insulator. The use of the internal degree of freedom of a bound half-fluxon pair was proposed there to test the time dilation. It was found that internal oscillations of the bound pair can play the role of a clock. ${ }^{16}$ A Lorentz reduction in their frequency was numerically observed ${ }^{16}$ when the bound half-fluxon pair was moving with relativistic velocities. Here we demonstrate that, to observe the time-dilation effect there is no need to use the double sine-Gordon equation ${ }^{16}$ but the additional degree of freedom of the $(2+1)$ D SG equation can be used to realize the internal clock. Indeed, the shape excitation moving along a fluxon provides such a clock. In other words, the position of the excitation on a fluxon acts as a "minute hand." From Eq. (4) the position of a vortex changes with time as $y_{0}(t)$ $=v t$ while the position of the excitation is

$$
x_{0}(t)=\mp\left[t-v y_{0}(t)\right] /\left(1-v^{2}\right)^{1 / 2}=\mp t\left(1-v^{2}\right)^{1 / 2} .
$$

Thus, the excitation moves along $x$ with speed

$$
|u|=\left|\dot{x}_{0}\right|=\left(1-v^{2}\right)^{1 / 2}<1 .
$$

In other words, all dynamic processes related to a relativistic moving vortex are slowed down by a time-dilation factor $\left(1-v^{2}\right)^{1 / 2}$, as in special relativity, compared to the stationary vortex. Our numerical calculations confirm that the collision of two solitary waves is also slowed down, compared to the collision at the rest frame as in Fig. 2.

\section{SINE-TYPE RUNNING SHAPE WAVES}

Apart from solitary waves, there exist exact solutions from the class of Eq. (2) associated with running waves. It is well known that the 1D SG kink possesses a linear oscillation mode with frequency $\omega=0$, a zero mode (see, e.g., Ref. 19). Linearizing the $(2+1) \mathrm{D} \mathrm{SG}$ equation around the stationary SG kink solution would lead to the equation for the propagation of linear waves along it with some wave vector $k$ directed along the vortex. The small-amplitude waves running along a vortex are of the form of a zero-mode modulated along $y$. Choosing $f=A \sin (\omega t \pm k x)$, with an arbitrary real constant $A$, we immediately obtain the existence of electromagnetic waves with a linear dispersion law $\omega= \pm k$. A similar linear-dispersion relation was obtained in the "sawtooth-Gordon" approximation, ${ }^{20}$ for linear waves on an array of Josephson vortices ${ }^{21}$ and single vortices. ${ }^{14}$ In contrast to the results in Refs. 14, 20, and 21 which were obtained using approximations, Eq. (2) allows to explicitly write the exact solution

$$
\varphi(x, y, t)=4 \arctan \exp [y-A \sin (\omega t \pm k x)]
$$

of the $(2+1)$ D SG equation corresponding to waves propagating along a vortex. This exact solution is valid for any amplitude $A$ with $\omega= \pm k$. It is worth analyzing the effect of the Lorentz invariance on the running waves Eq. (5). Surprisingly, an harmonic excitation $f=A \sin (\omega t \pm k x)$ propagating along a stationary vortex, Eq. (5), for a relativisticallymoving vortex becomes an asymmetric sawtoothlike function, as shown in Fig. 6.
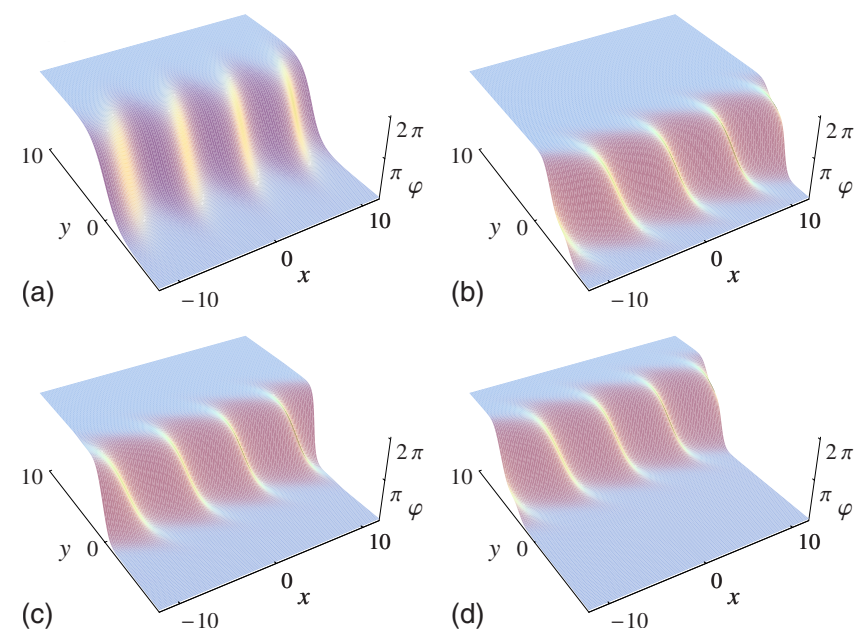

FIG. 6. (Color online) (a) Harmonic waves $f=A \sin (\omega t-k x)$ with $A=1$ and $\omega=k=1$ propagating along a stationary vortex according the exact solution Eq. (5) of the $2+1$ sine-Gordon equation. $[(\mathrm{b}),(\mathrm{c})$, and (d)] Harmonic waves propagating along a vortex moving with the speed $v=0.8$ along the $y$ axis (toward top left), as follows from the Lorentz transformation Eq. (4) of the solution Eq. (5). Figures (b), (c), and (d) correspond to the times $t=-5, t=0$, and $t=5$, respectively. The phase and group velocity of waves on a moving vortex are suppressed by the factor $\left(1-v^{2}\right)^{1 / 2}$. The processes on a moving vortex are slowed due to a relativistic timedilation effect, which is analogous to that of special relativity. The propagating harmonic excitation $f(z)=\sin (z)$ becomes significantly distorted to a sawtooth function due to the relativistic effect.

For waves on a moving vortex, the dispersion law changes to $\omega= \pm k\left(1-v^{2}\right)^{1 / 2}$. Indeed, the dispersion law for the waves can be found as follows. The phase velocity $\omega / k$ coincides with the speed of the excitation, $\left(1-v^{2}\right)^{1 / 2}$, which is universal for excitations of any shape. Using Eq. (5) and the position of a vortex line $y_{0}(t)=v t$, we obtain the phase velocity of the wave, $\omega / k= \pm\left(1-v^{2}\right)^{1 / 2}$. From this follows the dispersion law

$$
\omega= \pm k\left(1-v^{2}\right)^{1 / 2}
$$

This property could perhaps be used to control the dispersion of the waves produced by moving Josephson vortices.

\section{SHAPE WAVES OF A KINK PROFILE: PROPOSAL FOR EXPERIMENTS}

Experimentally, shape waves can be excited when a fluxon line interacts with inhomogeneities introduced on its path. When a vortex line passes through a curved or shaped region of a Josephson junction, the shape distortions of a vortex line are excited by inhomogeneities of the boundary. The shape of the excited waves is determined by the shape of the inhomogeneity as well as the vortex velocity. Below we present our analytical and numerical results for the case of a vortex line interacting with a barrier formed by a Josephson junction with a locally increased width, as shown in Fig. 7 (a). Another intriguing possibility to generate the shape 

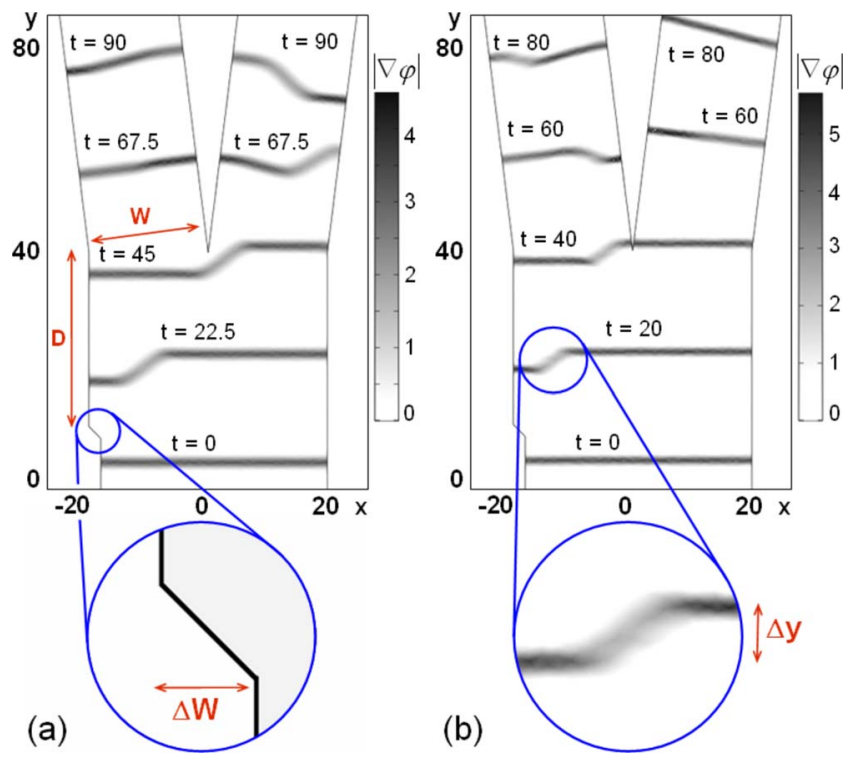

FIG. 7. (Color online) Detection of a vortex shape excitation and measurement of its speed in a $\mathrm{Y}$ junction with a small span angle. The figure shows snapshots of the superconducting phase gradient $|\nabla \varphi|$ obtained by numerically solving the $(2+1) \mathrm{D}$ SG equation in a $\mathrm{Y}$ junction geometry of size $40 \times 80 \lambda_{J}^{2}$ with Neumann boundary conditions: $\mathbf{n} \cdot \nabla \varphi=0$. At time $t=0$, a fluxon line moving with velocity (a) $v=0.8$ or (b) $v=0.9$ approaches a geometrical energy barrier (shown in the left inset). When passing the barrier, a shape excitation (zoomed in the right inset) is induced on the left end of a fluxon line and propagating to the right along the vortex. The propagating fluxon is split by the $\mathrm{Y}$ junction in two parts, one containing the shape excitation, and one without it. Depending on the velocity $v$ of the vortex line, the shape excitation can be directed either (a) to the right branch, if the velocity $v$ is low or (b) to the left branch, if $v$ is higher. This is due to the time dilation reducing the speed of the shape excitation. Measuring the critical velocity $v_{c}$ of the vortex line when such a transition occurs, the speed of a shape excitation can be found as $u=v_{c} W / D$. The presence of a shape excitation reduces the average velocity $\langle v\rangle$ of a vortex line. For instance, at $t=80$ in (b) the vortex in the left branch is noticeably delayed from the vortex in the right branch of the $\mathrm{Y}$ junction.

excitation is to use a $\mathrm{T}$ junction to create a cloning barrier ${ }^{22}$ on the path of a propagating fluxon line. Other types of inhomogeneities, besides these geometrical ones, such as microshorts or microresistors, realized by variations in the critical current (see, e.g., Ref. 5) can initiate shape excitations. One more possibility is using injectors of electric current connected locally to a Josephson junction ${ }^{23}$ where the fluxon line is propagating. This allows to conveniently control the shape of the excitations by varying the electric current of injectors.

In order to detect the propagation of a shape wave we propose the following scheme which involves the use of a Y-shaped cloning junction. ${ }^{22}$ Such junction can split a vortex into two parts, which afterward propagate independently from each other. For this purpose, a Y junction with a small span angle is preferable in order to minimize the disturbance on the vortex line. When a vortex with a propagating shape wave is split by such a junction, the shape excitation is directed to one of the branches of the Y junction, see Fig. 7. A shape wave can be generated from a boundary by locally increasing the width of the Josephson junction, as shown in the inset of Fig. 7(a). Because of the associated energy barrier, one end of the fluxon line is delayed with respect to the other one, which results in a kink profile, as shown in Fig. 7. The generated kink starts to propagate along the vortex line away from the boundary, carrying information about the induced delay to the rest of the vortex.

Consider now an arbitrary-shape excitation with function $f$ satisfying $f(-W) \rightarrow-\Delta f / 2, f(W) \rightarrow \Delta f / 2$ for $W \rightarrow \infty$. The total energy of this shape excitation is the difference of the energy $E[\varphi]$ of the solution Eq. (4) and the energy $E[\varphi]_{f \equiv 0}$ of the vortex without excitation: $\Delta E=E[\varphi]-E[\varphi]_{f \equiv 0}$. We obtain the following analytic expression for the total energy (see Appendix B)

$$
\Delta E=\frac{8}{\sqrt{1-v^{2}}}\left[\int_{-\infty}^{\infty} f^{\prime}(x)^{2} d x-v \Delta f\right],
$$

which is exact in the limit $W \rightarrow \infty$ and valid for an arbitrary excitation $f(\xi)$ of height $\Delta f$. As seen from Eq. (6), the energy $\Delta E$ can take negative values if $f$ is a kink $(\Delta f \neq 0)$ propagating along a moving vortex line. Indeed, this is in agreement with our numerical simulations (Fig. 7) which indicate that the increased width $\Delta W$ of the Josephson junction is compensated by the energy gained due to the propagating kink with negative $\Delta E$. Neglecting radiation losses, we now write the conservation of energy relation for a moving vortex line

$$
\Delta E+8 \Delta W\left(1-v^{2}\right)^{-1 / 2}=0 .
$$

Using Eq. (6), the minimal size of the kink can be estimated

$$
\Delta f \geq \Delta f_{\min }(\Delta W, v)=\Delta W / v .
$$

Note, that the apparent height $\Delta y$ of a kink propagating along a moving vortex line is Lorentz contracted, $\Delta y=\Delta f \sqrt{1-v^{2}}$. To describe the kinks in Fig. 7 we use the approximation $f(\xi)=(1 / 2) \Delta f \tanh (2 v \xi / \Delta f)$. Substituting it in Eq. (6) we obtain

$$
\Delta E=-8 \Delta f v\left(1-v^{2}\right)^{-1 / 2} / 3 .
$$

From energy conservation, we calculate the apparent height of the kink to be

$$
\Delta y=3 \sqrt{1-v^{2}} \Delta W / v .
$$

Substituting the value $\Delta W=2$, we obtain the following results for the apparent heights of the kinks: $\Delta y \simeq 4.5$ for $v$ $=0.8$ and $\Delta y \simeq 2.9$ for $v=0.9$, which agree with the numerical results shown in Figs. 7(a) and 7(b), respectively.

After passing the $\mathrm{Y}$ junction, each half of the initial vortex line is directed to a Josephson junction of width $W$. The presence of the shape kink slows down the average speed of a vortex line, see Appendix C

$$
\langle v\rangle=-\frac{1}{2 \pi W} \iint \dot{\varphi} d x d y=v-\frac{\Delta y \sqrt{1-v^{2}}}{W} .
$$

Thus, the presence of a shape wave can be directly detected by measuring the voltage on the Josephson junction, proportional to $\langle v\rangle$. A convenient system to study shape excitations would be a $\mathrm{Y}$ junction introduced into a ring, forming a 
$\sigma$-shaped Josephson junction which has been recently realized experimentally with high- $T_{c}$ superconductors in the context of generation of terahertz waves. ${ }^{24}$

The effect of time dilation is to slow down the shape excitation when the vortex line reaches relativistic velocities. If the velocity of the shape excitation is smaller than some critical value, it will be directed to the left branch of the Y junction, as shown in the Fig. 7(b), while the vortex propagating in the right branch remains undisturbed. However, if the shape excitation propagates faster, it will be directed to the right branch, Fig. 7(a). Because of the change in the average velocity Eq. (7) of a vortex line, a voltage step, corresponding to a shape wave changing the branch of the Y junction, will be exhibited on the $I-V$ characteristics. Finding the velocity $v_{c}$ of a fluxon line from the position of the voltage step, the speed of the shape excitation can be calculated as

$$
u=v_{c} W / D,
$$

where $D$ is the distance between the barrier and the $\mathrm{Y}$ junction, see Fig. 7. In this way, the relativistic factor $\left(1-v^{2}\right)^{1 / 2}$ for the delay of the shape kink propagation can be tested.

In an underdamped Josephson junction, a fluxon can be accelerated to velocities close to the Swihart velocity, typically on the order of $10^{7} \mathrm{~m} / \mathrm{s}$ for $\mathrm{Nb}$ Josephson junctions. The velocity of a fluxon depends both on the damping and the applied current. ${ }^{5}$ For a damping parameter $\alpha=0.01$ and current $\gamma=0.03$, in normalized units, ${ }^{5}$ it is possible to accelerate a vortex to the velocity $v \simeq 0.92$, so that the relativistic time-dilation factor would be $\left(1-v^{2}\right)^{1 / 2} \simeq 0.4$. For instance, for typical $\mathrm{Nb} / \mathrm{Al}-\mathrm{AlO}_{x} / \mathrm{Nb}$ Josephson junctions with a plasma frequency $\omega_{p} / 2 \pi \sim 50 \mathrm{GHz}$, the propagation time of the shape excitation between the two ends of a vortex line in a configuration as in Fig. 7 would be approximately $0.4 \mathrm{~ns}$ for a stationary vortex, while for a vortex moving with velocity $v=0.92$ this value would be relativistically delayed by $0.6 \mathrm{~ns}$.

\section{WOBBLING WAVES OF A JOSEPHSON VORTEX}

We have studied the effect of nonlinearity in the propagation of waves along a Josephson vortex. Here we focus on finding exact solutions and we intentionally do not address the issue of the stability of these waves, as this would require a separate work. The exact solutions are found by Lorentz transform in a second $(y)$ coordinate of the $N$-soliton solutions of the $(1+1) \mathrm{D}$ sine-Gordon equation. The $N$-soliton solutions are usually obtained by the Backlund transformation or the inverse scattering method. The three-soliton solution can be seen as a nonlinear superposition of a kink and a breather. ${ }^{3,4}$ Such solutions have been discussed earlier in the literature in the context of wobbling modes of a sine-Gordon soliton. ${ }^{25-27}$ The $(1+1) \mathrm{D}$ soliton wobbling mode can be written in the following symmetric form

$$
\varphi(x, t)=4 \arctan \left[\frac{e^{x} \cosh \left(\nu-\frac{\theta}{2}\right) \cosh \left(x \sqrt{1-\omega^{2}}+\nu+\frac{\theta}{2}\right)-\frac{\sqrt{1-\omega^{2}}}{\omega} \cosh \left(\nu+\frac{\theta}{2}\right) \cos \omega t}{\cosh \left(\nu+\frac{\theta}{2}\right) \cosh \left(x \sqrt{1-\omega^{2}}+\nu-\frac{\theta}{2}\right)-e^{x} \frac{\sqrt{1-\omega^{2}}}{\omega} \cosh \left(\nu-\frac{\theta}{2}\right) \cos \omega t}\right]
$$

where $\theta$ is given by

$$
\theta=\log \left(\frac{1-\sqrt{1-\omega^{2}}}{1+\sqrt{1-\omega^{2}}}\right) .
$$

Here the function $\arctan ()$ is not limited to the principal branch but defined as $\arctan (b / a)=\operatorname{Im}[\log (a+i b)]$. The solution Eq. (8) can be verified by direct substitution to the $(1+1) \mathrm{D}$ sine-Gordon equation and using computerized algebra. Equation (8) is a nonlinear superposition of a kink located at $x=0$ and a breather with its position parametrized by $\nu$ and given by $x_{\text {breather }}$ $\simeq-(\nu-\theta / 2) / \sqrt{1-\omega^{2}}$. Changing $\nu$ shifts the position of the breather with respect to the kink. Thus, there exist an infinite number of such solutions for a given frequency $\omega \in(0,1)$ : a family of solutions parametrized by $\nu \in(-\infty, \infty)$. A dense set of nonlinear wobbles fills the whole range of frequencies between 0 and 1 without gap in the spectrum.

Performing the Lorentz transformation on Eq. (8), we get 


$$
\varphi(x, y, t)=4 \arctan \left[\frac{e^{x} \cosh \left(\nu-\frac{\theta}{2}\right) \cosh \left(x \sqrt{1-\omega^{2}}+\nu+\frac{\theta}{2}\right)-\frac{\sqrt{1-\omega^{2}}}{\omega} \cosh \left(\nu+\frac{\theta}{2}\right) \cos (\Omega t-k y)}{\cosh \left(\nu+\frac{\theta}{2}\right) \cosh \left(x \sqrt{1-\omega^{2}}+\nu-\frac{\theta}{2}\right)-e^{x} \frac{\sqrt{1-\omega^{2}}}{\omega} \cosh \left(\nu-\frac{\theta}{2}\right) \cos (\Omega t-k y)}\right] .
$$

As seen from Eq. (9), a Lorentz transformation in the $y$ coordinate introduces a periodic modulation in $y$. Such a solution is localized in a finite area of $x$ and represents a wave propagating along $y$ with renormalized frequency $\Omega$ $=\sqrt{\omega^{2}+k^{2}}$. Such waves can be called wobbling waves because they originated from the $(1+1) \mathrm{D}$ soliton wobbling mode Eq. (8). Obviously, in the special case $k=0$ we recover the original wobbling solution Eq. (8). Note that the solution (9) is exact and nonperturbative.

Consider the limit $\omega \rightarrow 1$. In this case we can make a decomposition in Eq. (9) with $\sqrt{1-\omega^{2}} / \omega$ as a small parameter (for details, see Appendix A). This gives

$$
\begin{aligned}
\varphi(x, y, t) \simeq & 4 \arctan e^{x}+a\left[\frac{\tanh \nu-\tanh (a x / 4+\nu)}{\cosh x}\right. \\
& \left.+\frac{\tanh x \cos (\Omega t-k y)}{\cosh (a x / 4+\nu)}\right],
\end{aligned}
$$

where we have introduced the amplitude $a=4 \sqrt{1-\omega^{2}}$. From this follows the equation for the spectrum

$$
\Omega=\left(1+k^{2}-a^{2} / 16\right)^{1 / 2}
$$

for any $\nu$. The case $\nu=0$ corresponds to a wave propagating on a Josephson vortex. One can estimate the size of the domain $\Delta x$ where the wave is localized around the vortex

$$
\Delta x \simeq 4 / a=1 / \sqrt{1-\omega^{2}} .
$$

The nonzero $\nu$ correspond to waves shifted from a Josephson vortex to either side. For high $|\nu| \gg 1$, the wave separates from the Josephson vortex and persists in its vicinity at a distance $\simeq-4 \nu / a=-\nu / \sqrt{1-\omega^{2}}$. In contrast to the case of linear waves, the nonlinear ones may exist even without the presence of a Josephson vortex; although, the effect of a Josephson vortex to their properties is crucial. As seen from Eq. (10), the effect of a vortex is the reversed symmetry of the wave: the term $\tanh x$ in Eq. (10) makes it asymmetric.

Let us focus attention to a wave running along a Josephson vortex. From Eq. (9), the exact solution for $\nu=0$ is

$$
\varphi(x, y, t)=4 \arctan \left[\frac{e^{x} \cosh \left(x \sqrt{1-\omega^{2}}+\frac{\theta}{2}\right)-\frac{\sqrt{1-\omega^{2}}}{\omega} \cos (\Omega t-k y)}{\cosh \left(x \sqrt{1-\omega^{2}}-\frac{\theta}{2}\right)-e^{x} \frac{\sqrt{1-\omega^{2}}}{\omega} \cos (\Omega t-k y)}\right] .
$$

The cross section of this wave at different oscillation phases is shown in Fig. 8. Figure 9 shows a snapshot at $t=0$ of wobbling wave with $k=0.8$ and $\omega=0.9$. For comparison, we present graphs corresponding to the waves shifted with respect to the vortex, see Figs. 10-13. For small values of the amplitude $a=4 \sqrt{1-\omega^{2}}$ of oscillation, we can write

$$
\begin{aligned}
\varphi(x, y, t) & \simeq 4 \arctan e^{x}+\frac{4 \sqrt{1-\omega^{2}} \cos (\Omega t-k y)}{\cosh \left(x \sqrt{1-\omega^{2}}\right)} \tanh x \\
& =4 \arctan e^{x}+a \frac{\cos (\Omega t-k y)}{\cosh (a x / 4)} \tanh x .
\end{aligned}
$$

Using Eq. (12) we estimate the size of the domain $\Delta x$ where the wave is localized around the vortex, $\Delta x \simeq 4 / a$. This is similar to the low amplitude breathers existing without the presence of a vortex or away from it. After making a Lorentz transform in the $y$ coordinate of a sine-Gordon breather and

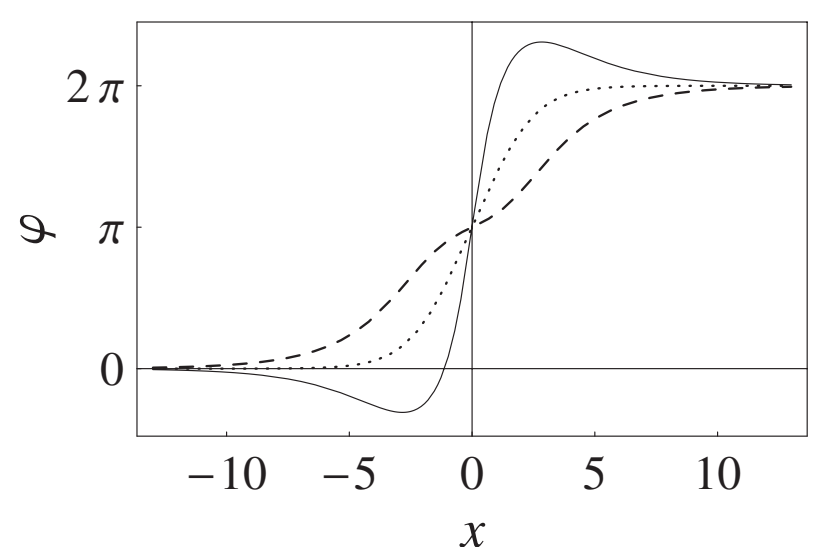

FIG. 8. Cross section of wobbling wave with $\nu=0$. The solid line corresponds to $\cos (\Omega t-k y)=1$, the dashed line corresponds to $\cos (\Omega t-k y)=-1$, and the dotted line to $\cos (\Omega t-k y)=0$. 


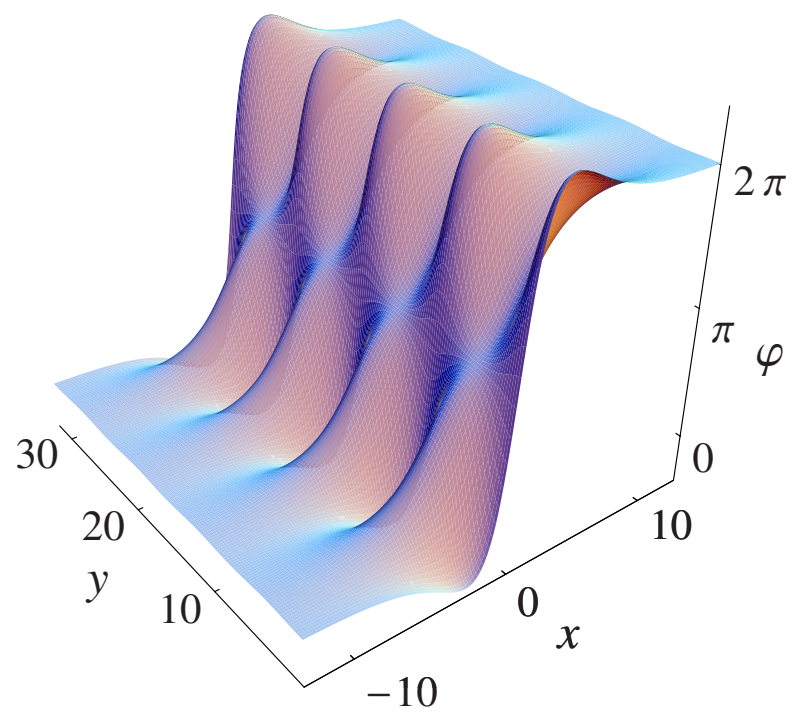

FIG. 9. (Color online) Snapshot of wobbling wave with $\nu=0$ described by Eq. (9).

making a decomposition in a similar fashion, we obtain

$$
\varphi(x, y, t) \simeq a \frac{\cos (\Omega t-k y)}{\cosh (a x / 4)}
$$

and $\Omega=\left(1+k^{2}-a^{2} / 16\right)^{1 / 2}$. Such waves exist in some distance from the Josephson vortex and are described by Eq. (9) in the limit $\nu \gg \theta / 2+\sqrt{1-\omega^{2}}$. As seen from Eq. (10), the effect of a vortex on a propagating wobbling waves is peculiar: the term tanh $x$ in Eq. (10) results in an antisymmetric wave in its cross section, e.g., compare Fig. 8 versus Figs. 10 and 12.

\section{DISCUSSION}

The class of solutions Eq. (4) is important for describing the interaction of moving Josephson vortices with pinning centers inside the Josephson junction or in the boundary. The interaction of a Josephson vortex line with inhomogeneities results in an excitation of the type, Eq. (4).

We have predicted the existence of excitations of arbitrary shape, propagating along stationary or moving Josephson

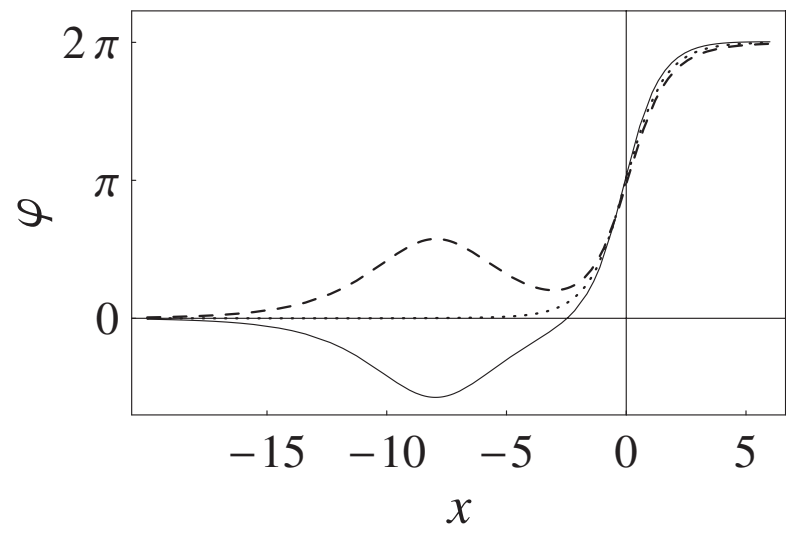

FIG. 10. Cross section of wobbling wave with $\nu=3$. The solid line corresponds to $\cos (\Omega t-k y)=1$, the dashed line corresponds to $\cos (\Omega t-k y)=-1$, and the dotted line to $\cos (\Omega t-k y)=0$.

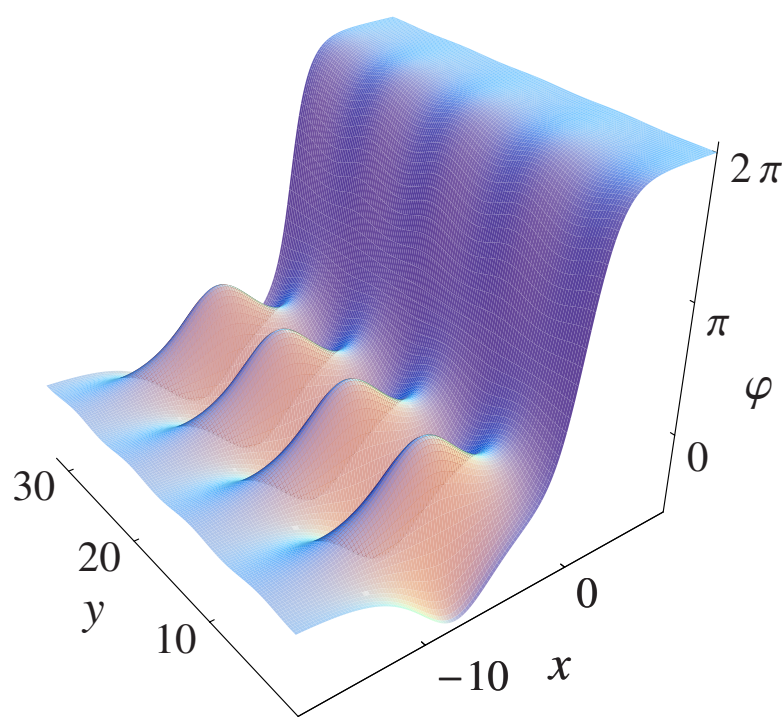

FIG. 11. (Color online) Snapshot of wobbling wave with $\nu=3$ described by Eq. (9).

vortices in 2D Josephson junctions, and obtained exact solutions of the $(2+1)$ D SG equation. Remarkably, for some conditions, a shape wave can carry negative energy, i.e., a vortex with a shape excitation can have smaller energy than the same vortex without it. Based on our analysis, we suggest how to test a time-dilation effect analogous to that in special relativity using the shape excitation as a minute hand measuring the proper time of the vortex coordinate frame.

Apart from the shape waves originated from the shift mode of a Josephson vortex line, a different class of nonlinear waves exist in 2D Josephson junctions, which we call here wobbling waves. These correspond to the higher mode of the excitation of a soliton, a wobbling mode.

\section{ACKNOWLEDGMENTS}

We acknowledge partial support from the National Security Agency (NSA), Laboratory for Physical Sciences (LPS), U.S. Army Research Office (USARO), the National Science

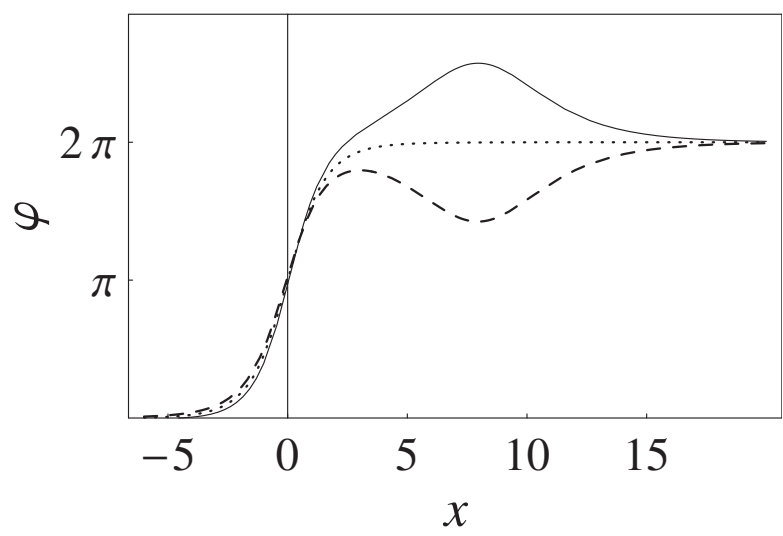

FIG. 12. Cross section of wobbling wave for $\nu=-3$. Solid line corresponds to $\cos (\Omega t-k y)=1$, dashed line corresponds to $\cos (\Omega t-k y)=-1$, and dotted line to $\cos (\Omega t-k y)=0$. 


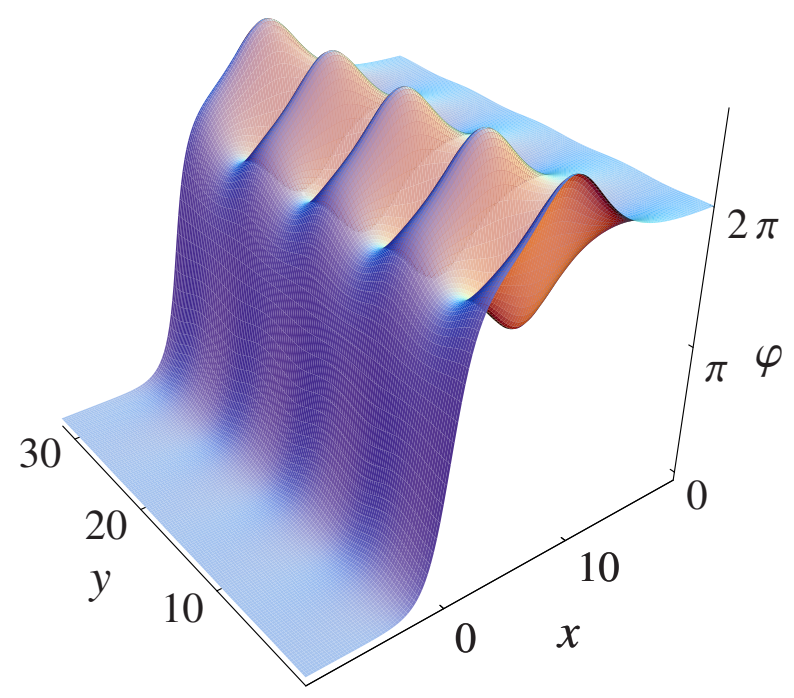

FIG. 13. (Color online) Snapshot of wobbling wave with $\nu=-3$ described by Eq. (9).

Foundation (NSF) under Grant No. EIA-0130383, support from JSPS under Contract No. RFBR 06-02-91200, and Core-to-Core (CTC) program supported by JSPS. Also, support from U.K. EPSRC via Grants No. EP/D072581/1, No. EP/E042589/1, and No. EP/F005482/1, and ESF network program "Arrays of Quantum Dots and Josephson Junctions." S.S. acknowledges support from the Ministry of Education, Science, Culture and Sport of Japan via the Grant-inAid for Young Scientists Grant No. 18740224.

\section{APPENDIX A: EXACT SOLUTIONS FOR WAVES OF ARBITRARY SHAPE}

The $(2+1)$ D sine-Gordon equation Eq. (1) reads

$$
\varphi_{t t}-\varphi_{x x}-\varphi_{y y}+\sin \varphi=0 .
$$

Let us now consider the special case $f \equiv 0$, a stationary sine-Gordon soliton without shape excitation,

$$
\varphi(x, y, t)=4 \arctan e^{y},
$$

is a well-known solution of Eq. (13) (see, e.g., Ref. 4). Indeed,

$$
\begin{gathered}
\varphi_{t t}=0, \\
\varphi_{x x}=0, \\
\varphi_{y}=2 \operatorname{sech} y, \quad \varphi_{y y}=-2 \operatorname{sech} y \tanh y, \\
\sin \varphi=-2 \operatorname{sech} y \tanh y,
\end{gathered}
$$

which proofs that the sine-Gordon soliton, Eq. (14), is a solution of Eq. (13). Let us now consider a more general situation when the propagating shape excitation is present on a vortex and now substitute the expression

$$
\varphi(x, y, t)=4 \arctan \exp [y-F(x, t)]
$$

into Eq. (13). Similarly as before, we now explicitly write all the relevant terms

$$
\begin{gathered}
\varphi_{t t}=-2 \operatorname{sech}[y-F(x, t)]\left\{\tanh [y-F(x, t)]\left(\frac{\partial F}{\partial t}\right)^{2}+\frac{\partial^{2} F}{\partial t^{2}}\right\}, \\
\varphi_{x x}=-2 \operatorname{sech}[y-F(x, t)]\left\{\tanh [y-F(x, t)]\left(\frac{\partial F}{\partial x}\right)^{2}+\frac{\partial^{2} F}{\partial x^{2}}\right\}, \\
\varphi_{y y}=-2 \operatorname{sech}[y-F(x, t)] \tanh [y-F(x, t)], \\
\sin \varphi=-2 \operatorname{sech}[y-F(x, t)] \tanh [y-F(x, t)] .
\end{gathered}
$$

Thus Eq. (15) satisfies the Eq. (13), if the function $F$ satisfies the following system of equations

$$
\left\{\begin{array}{l}
\left(\frac{\partial F}{\partial t}\right)^{2}=\left(\frac{\partial F}{\partial x}\right)^{2} \\
\frac{\partial^{2} F}{\partial t^{2}}=\frac{\partial^{2} F}{\partial x^{2}}
\end{array} .\right.
$$

Indeed, choosing $F(x, t)=f(x \pm t)$, as in Eq. (2), with an arbitrary real-valued twice-differentiable function $f(\xi)$, satisfies both Eq. (16) simultaneously.

\section{APPENDIX B: ENERGY OF THE SHAPE KINK}

The total energy of the two-dimensional Josephson junction is defined by the sine-Gordon Hamiltonian

$E[\varphi]=\int_{-\infty}^{\infty} d y \int_{-W}^{W} d x\left[\frac{\varphi_{t}^{2}}{2}+\frac{\varphi_{x}^{2}}{2}+\frac{\varphi_{y}^{2}}{2}+1-\cos \varphi\right]$,

where the integration is over the area of a Josephson junction in the limit of a long contact of width $2 W \gg 1$. Substituting

$$
\varphi_{0}(x, y, t)=4 \arctan \exp \left(\frac{y-v t}{\sqrt{1-v^{2}}}\right)
$$

and integrating over $x$, we obtain the energy of a moving Josephson vortex line without excitation

$E\left[\varphi_{0}\right]=\frac{4}{1-v^{2}} \int_{-\infty}^{\infty} d y \int_{-W}^{W} d x \operatorname{sech}\left(\frac{y}{\sqrt{1-v^{2}}}\right)^{2}=\frac{16 W}{\sqrt{1-v^{2}}}$

Substituting Eq. (4) of the paper,

$\varphi(x, y, t)=4 \arctan \exp \left[\frac{y-v t}{\sqrt{1-v^{2}}}-f\left(x \pm \frac{t-v y}{\sqrt{1-v^{2}}}\right)\right]$,

into the expression for the energy, Eq. (17), we obtain

$$
\begin{aligned}
E[\varphi]= & \frac{4}{1-v^{2}} \int_{-\infty}^{\infty} d y \int_{-W}^{W} d x \text { sech } \\
& \times\left[\frac{y-v t}{\sqrt{1-v^{2}}}-f\left(x \pm \frac{t-v y}{\sqrt{1-v^{2}}}\right)\right]^{2} \\
& \times\left[1-2 v f^{\prime}\left(x \pm \frac{t-v y}{\sqrt{1-v^{2}}}\right)+f^{\prime}\left(x \pm \frac{t-v y}{\sqrt{1-v^{2}}}\right)^{2}\right] .
\end{aligned}
$$

Taking $t=0$, changing the variable $y \rightarrow y \sqrt{1-v^{2}}$ and choos- 
ing, for example, the second sign $(-)$, we obtain

$E[\varphi]$

$$
=\frac{4}{\sqrt{1-v^{2}}} \int_{-\infty}^{\infty} d y \int_{-W}^{W} d x \frac{\left[1-2 v f^{\prime}(x+v y)+f^{\prime}(x+v y)^{2}\right]}{\cosh [y-f(x+v y)]^{2}} .
$$

Let the arbitrary function $f(\xi)$ be a kink

$$
f(\xi)=A f_{0}(\xi),
$$

where $f_{0}(\xi)$ is a unitary kink: an arbitrary function $f$ that satisfies $f_{0}(-W) \rightarrow-1, f_{0}(W) \rightarrow 1$ for $W \rightarrow \infty$, e.g., $f_{0}(\xi)$ $=\tanh \xi, f_{0}(\xi)=\tanh ^{3} \xi, f_{0}(\xi)=(-\pi+4 \arctan \exp \xi) / \pi$, etc. With this definition, the unitary kink $f_{0}(\xi)$ possesses the following properties

$$
\begin{gathered}
\int_{-W}^{W} d x f_{0}(x+v y)^{2 n+1}=2 v y, \\
\int_{-W}^{W} d x f_{0}(x+v y)^{2 n+1} f_{0}^{\prime}(x+v y)=\text { const, } \\
\int_{-W}^{W} d x f_{0}(x+v y)^{2 n+1} f_{0}^{\prime}(x+v y)^{2}=\text { const, } \\
\int_{-W}^{W} d x f_{0}(x+v y)^{2 n}=\text { const } \\
\int_{-W}^{W} d x f_{0}(x+v y)^{2 n} f_{0}^{\prime}(x+v y)=\text { const, } \\
\int_{-W}^{W} d x f_{0}(x+v y)^{2 n} f_{0}^{\prime}(x+v y)^{2}=\text { const }
\end{gathered}
$$

in the limit $W \rightarrow \infty$, where $n$ is an integer: $n=0,1,2, \ldots$ Let us now define the energy of the shape excitation as $\Delta E$ $\equiv E[\varphi]-E\left[\varphi_{0}\right]$. Substituting $E\left[\varphi_{0}\right]$ and $E[\varphi]$ from Eqs. (18) and (20),

$$
\begin{aligned}
\Delta E= & \frac{4}{\sqrt{1-v^{2}}} \int_{-\infty}^{\infty} d y \int_{-W}^{W} \\
& \times d x\left\{\frac{1-2 v A f_{0}^{\prime}(x+v y)+A^{2} f_{0}^{\prime}(x+v y)^{2}}{\cosh \left[y-A f_{0}(x+v y)\right]^{2}}\right. \\
& \left.-\frac{1}{\cosh ^{2} y}\right\}
\end{aligned}
$$

in the limit $W \rightarrow \infty$. In order to calculate this integral, we use a decomposition to the Taylor series in $A$,

$$
\frac{1}{\cosh \left[y-A f_{0}(x+v y)\right]^{2}}=\sum_{m=0}^{\infty}\left[-A f_{0}(x+v y)\right]^{m} \frac{d^{m}}{d y^{m}} \frac{1}{\cosh ^{2} y} .
$$

Substituting this series into the expression for $\Delta E$, Eq. (23), and using the properties of the unitary kink, Eq. (22), $m=0$ gives

$$
\begin{gathered}
\int_{-W}^{W} d x\left[-2 v A f_{0}^{\prime}(x+v y)+A^{2} f_{0}^{\prime}(x+v y)^{2}\right] \\
\quad=-4 v A+A^{2} \int_{-W}^{W} d x f_{0}^{\prime}(x)^{2}
\end{gathered}
$$

and $m=1$ gives

$$
\begin{aligned}
& \int_{-W}^{W} d x\left[-A f_{0}(x+v y)\right]\left[1-2 v A f_{0}^{\prime}(x+v y)+A^{2} f_{0}^{\prime}(x+v y)^{2}\right] \\
& \quad=-2 A v y .
\end{aligned}
$$

Using the unitary kink properties, Eq. (22) for integrating the terms $m \geq 2$ over $x$, and then integrating them over $y$, the terms $m \geq 2$ contain

$$
\int_{-\infty}^{\infty} \frac{d^{m}}{d y^{m}} \frac{1}{\cosh ^{2} y}=0 .
$$

Integrating by parts over $y$ and collecting the terms together, we obtain

$$
\Delta E=\frac{8 A}{\sqrt{1-v^{2}}}\left[A \int_{-\infty}^{\infty} f_{0}^{\prime}(x)^{2} d x-2 v\right] .
$$

Introducing the height of the kink $\Delta f=2 A$, and using Eq. (21), we obtain the expression for its energy, Eq. (6) of the paper,

$$
\Delta E=\frac{8}{\sqrt{1-v^{2}}}\left[\int_{-\infty}^{\infty} f^{\prime}(x)^{2} d x-v \Delta f\right]
$$

which is universal and valid for an arbitrary-shape excitation $f(\xi)$ of height $\Delta f$. For instance, choosing

$$
f(\xi)=\frac{\Delta f}{2} \tanh \left(\frac{2 g \xi}{\Delta f}\right)
$$

which is a kink of slope $g>0$ and size $\Delta f$, we obtain

$$
\Delta E=\frac{8 \Delta f}{\sqrt{1-v^{2}}}\left(\frac{2}{3} g-v\right) .
$$

If a kink is generated by a barrier of height $\Delta W$, using the general formula (24) we write the equation for the energy conservation

$$
\begin{aligned}
& \frac{8}{\sqrt{1-v^{2}}}\left[\int_{-\infty}^{\infty} f^{\prime}(x)^{2} d x-v \Delta f\right] \\
& =-\frac{8 \Delta W}{\sqrt{1-v^{2}}}-\frac{8 v \Delta f}{\sqrt{1-v^{2}}} \leq-\frac{8 \Delta W}{\sqrt{1-v^{2}}}
\end{aligned}
$$

so that 


$$
\Delta f \geq \Delta f_{\min }(\Delta W, v)=\frac{\Delta W}{v},
$$

where $\Delta f_{\min }(\Delta W, v)$ is only defined by the barrier height $\Delta W$ and the velocity of the vortex line $v$. This expression can be used to estimate the minimal height of the kink when neither the exact shape of the barrier nor the kink profile are known.

The apparent profile $\bar{y}(x)$ of the shape excitation at each moment of time $t$ can be found from Eq. (19) by solving the equation

$$
\frac{\bar{y}(x)-v t}{\sqrt{1-v^{2}}}=f\left[x \pm \frac{t-v \bar{y}(x)}{\sqrt{1-v^{2}}}\right]
$$

by means of progressive approximations. For $t=0$ and choosing, e.g., one sign (-), we find

$$
\bar{y}(x)=\sqrt{1-v^{2}} f\{x+v f[x+v f(\ldots)]\} .
$$

Taking the limit $x \rightarrow \pm \infty$, we find that the apparent height $\Delta y$ of the kink propagating along a moving vortex line is Lorentz contracted

$$
\Delta y=\Delta f \sqrt{1-v^{2}} .
$$

\section{APPENDIX C: AVERAGE VELOCITY OF A VORTEX LINE}

The presence of a shape kink may change the average speed of a vortex line. The average velocity for a vortex line moving in a Josephson junction of width $2 W$ is

$$
\langle v\rangle=-\frac{1}{4 \pi W} \int_{-\infty}^{\infty} d y \int_{-W}^{W} d x \dot{\varphi},
$$

where we integrate over the area of a Josephson junction of width $W$. Substituting Eq. (19) at $t=0$,

$$
\langle v\rangle=\frac{1}{2 \pi W} \int_{-\infty}^{\infty} d y \int_{-W}^{W} d x \frac{\left[v-f^{\prime}(x+v y)\right]}{\cosh [y-f(x+v y)]} .
$$

For $f \equiv 0$ we get $\langle v\rangle=v$, as expected. Consider the case when $f$ is an antisymmetric kink, $f(\xi)=A f_{0}(\xi)$. Similar to the cal- culation above, we use a decomposition to the Taylor series in $A$

$$
\frac{1}{\cosh \left[y-A f_{0}(x+v y)\right]}=\sum_{m=0}^{\infty}\left[-A f_{0}(x+v y)\right]^{m} \frac{d^{m}}{d y^{m}} \frac{1}{\cosh y},
$$

and use the properties, Eq. (22), of the unitary kink. The term $m=0$ gives

$$
\int_{-W}^{W} d x\left[v-A f_{0}^{\prime}(x+v y)\right]=2 W v-2 A .
$$

The term $m=1$ gives

$$
\int_{-W}^{W} d x\left[v-A f_{0}^{\prime}(x+v y)\right]\left[-A f_{0}(x+v y)\right]=-2 A v^{2} y .
$$

Integrating by parts and collecting the terms together, we obtain

$$
\langle v\rangle=v-\frac{A\left(1-v^{2}\right)}{W}=v-\frac{\Delta f\left(1-v^{2}\right)}{2 W} .
$$

After passing the $\mathrm{Y}$ junction, a half vortex line is directed to a Josephson junction of width $W$, which is twice smaller. In this case

$$
\langle v\rangle=v-\frac{\Delta f\left(1-v^{2}\right)}{W}=v-\frac{\Delta y \sqrt{1-v^{2}}}{W} .
$$

This is formula (7) for the average velocity $\langle v\rangle$ which we use in this paper.

\section{APPENDIX D}

Let us make a decomposition in Eq. (9) with a small parameter $\sqrt{1-\omega^{2}} / \omega \rightarrow 0$. Obviously, for $x \leq 0$ we can make the straightforward decomposition of Eq. (9). In the case $x$ $\geq 0$, the decomposition looks tricky because the second term in the denominator $e^{x} \sqrt{1-\omega^{2}} / \omega$ exceeds the first one in the limit $x \rightarrow+\infty$. To grasp the correct asymptotics at $x \rightarrow+\infty$ we need to modify the decomposing formula multiplying the denominator and nominator by $e^{-x}$ and using the identity $\arctan (z)=\pi / 2-\operatorname{arccot}(z)$. Obviously, the function

$$
\varphi(x, y, t)=2 \pi-4 \arctan \left[\frac{e^{-x} \cosh \left(\nu+\frac{\theta}{2}\right) \cosh \left(x \sqrt{1-\omega^{2}}+\nu-\frac{\theta}{2}\right)-\frac{\sqrt{1-\omega^{2}}}{\omega} \cosh \left(\nu-\frac{\theta}{2}\right) \cos (\Omega t-k y)}{\cosh \left(\nu-\frac{\theta}{2}\right) \cosh \left(x \sqrt{1-\omega^{2}}+\nu+\frac{\theta}{2}\right)-e^{-x} \frac{\sqrt{1-\omega^{2}}}{\omega} \cosh \left(\nu+\frac{\theta}{2}\right) \cos (\Omega t-k y)}\right]
$$

is equivalent to Eq. (9) but now can be decomposed in powers of $\sqrt{1-\omega^{2}} / \omega$ with the correct asymptotics at $x \rightarrow+\infty$. Both cases, $x \geq 0$ and $x \leq 0$, lead to the identical decomposition Eq. (10). 
${ }^{1}$ D. L. Mills, Nonlinear Optics (Springer, Berlin, 1998); Yu. S. Kivshar and B. A. Malomed, Rev. Mod. Phys. 61, 763 (1989).

${ }^{2}$ A. Barone and G. Paternó, Physics and Applications of Josephson Effect (Wiley, New York, 1982); Nonlinear Superconducting Devices and High-Tc Materials, edited by R. D. Parmentier and N. F. Pedersen (World Scientific, Singapore, 1995).

${ }^{3}$ P. G. Drazin and R. S. Johnson, Solitons: An Introduction (Cambridge University Press, Cambridge, 1989); E. A. Jackson Perspectives in Nonlinear Dynamics (Cambridge University Press, Cambridge, 1990).

${ }^{4}$ R. Rajaraman, Solitons and Instantons (Elsevier, Amsterdam, 1982).

${ }^{5}$ D. W. McLaughlin and A. C. Scott, Phys. Rev. A 18, 1652 (1978).

${ }^{6}$ D. R. Gulevich and F. V. Kusmartsev, Phys. Rev. B 74, 214303 (2006).

${ }^{7}$ I. L. Bogolyubskii and V. G. Makhankov, JETP Lett. 25, 12 (1976); 25, 107 (1977); P. L. Christiansen and O. H. Olsen, Phys. Lett. 68A, 185 (1978); P. L. Christiansen and P. S. Lomdahl, Physica D 2, 482 (1981); B. Piette and W. J. Zakrzewski, Nonlinearity 11, 1103 (1998); P. L. Christiansen, Niels Grønbech-Jensen, Peter S. Lomdahl, and Boris A. Malomed, Phys. Scr. 55, 131 (1997); X.-Y. Tang, S.-Y. Lou, and Y. Zhang, Phys. Rev. E 66, 046601 (2002); S.-Y. Lou, J. Phys. A 36, 3877 (2003).

${ }^{8}$ S. G. Lachenmann, G. Filatrella, T. Doderer, J. C. Fernandez, and R. P. Huebener, Phys. Rev. B 48, 16623 (1993); J. G. Caputo, N. Flytzanis, Y. Gaididei, and E. Vavalis, Phys. Rev. E 54, 2092 (1996); C. Gorria, Yu. B. Gaididei, M. P. Soerensen, P. L. Christiansen, and J. G. Caputo, Phys. Rev. B 69, 134506 (2004).

${ }^{9}$ B. G. Konopelchenko, W. K. Schief, and C. Rogers, Phys. Lett. A 172, 39 (1992); W. K. Schief, J. Phys. A 25, L1351 (1992); J. J. Nimmo, Phys. Lett. A 168, 113 (1992); J. J. C. Nimmo and W. K. Schief, Proc. R. Soc. London, Ser. A 453, 255 (1997); W. K. Schief, ibid. 453, 1671 (1997); S.-Y. Lou, C.-L. Chen, and X.-Y. Tang, J. Math. Phys. 43, 4078 (2002).

${ }^{10}$ S.-Y. Lou, J. Math. Phys. 41, 6509 (2000).

${ }^{11}$ D. R. Gulevich, F. V. Kusmartsev, S. Savel'ev, V. A. Yampol'skii, and F. Nori, Phys. Rev. Lett. 101, 127002 (2008).

${ }^{12}$ S. Savel'ev, A. L. Rakhmanov, and F. Nori, Phys. Rev. Lett. 98, 077002 (2007); S. Savel'ev, V. Yampol'skii, and F. Nori, ibid. 95, 187002 (2005); S. Savel'ev, A. Rakhmanov, V. Yampol'skii, and F. Nori, Nat. Phys. 2, 521 (2006); V. A. Yampol'skii, D. R.
Gulevich, S. Savel'ev, and F. Nori, Phys. Rev. B 78, 054502 (2008).

${ }^{13}$ V. P. Koshelets and S. V. Shitov, Supercond. Sci. Technol. 13, R53 (2000); V. P. Koshelets, A. B. Ermakov, L. V. Filippenko, A. V. Khudchenko, O. S. Kiselev, A. S. Sobolev, M. Y. Torgashin, P. A. Yagoubov, R. W. M. Hoogeveen, and W. Wild, IEEE Trans. Appl. Supercond. 17, 336 (2007); M. Yu. Torgashin, V. P. Koshelets, P. N. Dmitriev, A. B. Ermakov, L. V. Filippenko, and P. A. Yagoubov, ibid. 17, 379 (2007).

${ }^{14}$ D. R. Gulevich, S. Savel'ev, V. A. Yampol'skii, F. V. Kusmartsev, and F. Nori, J. Appl. Phys. 104, 064507 (2008).

${ }^{15}$ A. Matsuda and T. Kawakami, Phys. Rev. Lett. 51, 694 (1983); A. Laub, T. Doderer, S. G. Lachenmann, R. P. Huebener, and V. A. Oboznov, ibid. 75, 1372 (1995).

${ }^{16}$ M. Nishida, K. Murata, T. Fujii, and N. Hatakenaka, Phys. Rev. Lett. 99, 207004 (2007).

${ }^{17}$ D. Gupta, A. M. Kadin, R. J. Webber, I. Rochwarger, D. Bryce, W. J. Hollander, Young Uk Yim, Channakeshav, R. P. Kraft, Jin-Woo Kim, and J. F. McDonald, IEEE Trans. Appl. Supercond. 13, 477 (2003).

${ }^{18}$ Y. C. Fung, Foundations of Solid Mechanics (Prentice Hall, New Jersey, 1965); C. H. Scholz, The Mechanics of Earthquakes and Faulting (Cambridge University Press, Cambridge, 2002).

${ }^{19}$ J. A. González, A. Bellorín, and L. E. Guerrero, Phys. Rev. E 65, 065601(R) (2002).

${ }^{20}$ A. C. Scott, in The Application of Backlund Transform to Physical Problems, Lecture Notes in Mathematics Vol. 515, edited by R. Miura, (Springer, Berlin, New York, 1976), p. 80.

${ }^{21}$ I. O. Kulik, Sov. Phys. JETP 24, 1307 (1967); P. Lebwohl and M. J. Stephen, Phys. Rev. 163, 376 (1967); A. L. Fetter and M. J. Stephen, Phys. Rev. 168, 475 (1968); I. O. Kulik and I. K. Yanson, The Josephson Effect in Superconductive Tunneling Structures (Keter, Jerusalem, 1972).

${ }^{22}$ D. R. Gulevich and F. V. Kusmartsev, Phys. Rev. Lett. 97, 017004 (2006); Supercond. Sci. Technol. 20, S60 (2007); New J. Phys. 9, 59 (2007).

${ }^{23}$ A. V. Ustinov, Appl. Phys. Lett. 80, 3153 (2002); B. A. Malomed and A. V. Ustinov, Phys. Rev. B 69, 064502 (2004).

${ }^{24}$ D. R. Gulevich, M. Gaifullin, O. E. Kusmartseva, F. V. Kusmartsev, and K. Hirata, Physica C 468, 1903 (2008).

${ }^{25}$ H. Segur, J. Math. Phys. 24, 1439 (1983).

${ }^{26}$ G. Kälbermann, J. Phys. A 37, 11603 (2004).

${ }^{27}$ L. A. Ferreira, B. Piette, and W. J. Zakrzewski, Phys. Rev. E 77, 036613 (2008) 